\title{
Lepton Pair Čerenkov Radiation Emitted by Tachyonic Neutrinos: Lorentz-Covariant Approach and IceCube Data
}

\author{
Ulrich D. Jentschura ${ }^{1}$ and Robert Ehrlich ${ }^{2}$ \\ ${ }^{1}$ Department of Physics, Missouri University of Science and Technology, Rolla, MO 65409, USA \\ ${ }^{2}$ Department of Physics, George Mason University, Fairfax, VA 22030, USA \\ Correspondence should be addressed to Ulrich D. Jentschura; ulj@mst.edu
}

Received 22 February 2016; Revised 28 April 2016; Accepted 17 May 2016

Academic Editor: Theocharis Kosmas

Copyright (C) 2016 U. D. Jentschura and R. Ehrlich. This is an open access article distributed under the Creative Commons Attribution License, which permits unrestricted use, distribution, and reproduction in any medium, provided the original work is properly cited. The publication of this article was funded by SCOAP ${ }^{3}$.

\begin{abstract}
Current experiments do not exclude the possibility that one or more neutrinos are very slightly superluminal or that they have a very small tachyonic mass. Important bounds on the size of a hypothetical tachyonic neutrino mass term are set by lepton pair Čerenkov radiation (LPCR), that is, by the decay channel $\nu \rightarrow e^{+} e^{-} \nu$, which proceeds via a virtual $Z^{0}$ boson. Here, we use a Lorentz-invariant dispersion relation which leads to very tight constraints on the tachyonic mass of neutrinos; we also calculate decay and energy loss rates. A possible cutoff seen in the IceCube neutrino spectrum for $E_{v}>2 \mathrm{PeV}$, due to the potential onset of LPCR, is discussed.
\end{abstract}

\section{Introduction}

The early arrival of a neutrino burst from the 1987A supernova [1] still motivates speculations about a possible superluminal nature of neutrinos, even if it is generally assumed that the delay in the arrival of electromagnetic radiation (light) is caused by the time the shock wave from the core collapse needs in order to reach the surface of the exploding star. If neutrinos are ever so slightly superluminal, then they may emit Čerenkov radiation in the form of light lepton pairs. In this paper, we attempt to answer three questions: (i) How would the energy threshold for the decay channel $v \rightarrow e^{+} e^{-} v$ (lepton pair Čerenkov radiation, LPCR) have to be calculated if we assume a strictly Lorentz-covariant, space-like dispersion relation for the relevant neutrino flavor eigenstate? (ii) How would the decay rate and the energy loss rate have to be calculated under this assumption? Can the tachyonic Dirac equation [2-5] and its bispinor solutions $[6,7]$ be used in that context? (iii) What implications could be derived for astrophysics under the assumption that a possible cutoff seen by IceCube for neutrinos with energies $E_{v}>2 \mathrm{PeV}$ is confirmed by future experiments?

Theoretical arguments can be useful in restricting the possible degree of superluminality of neutrinos and maximum attainable neutrino velocities $[8-10]$. In $[8,9]$, a Lorentz-noncovariant dispersion relation $E_{\gamma}=|\vec{p}| v_{\gamma}$ was used, where $v_{v}>c$ is a constant parameter. This assumption leads to an energy-dependent effective "mass" square $E_{v}^{2}-$ $\vec{p}^{2} \approx E_{v}^{2}\left(v_{v}^{2}-1\right) v_{v}^{-2} \equiv m_{\mathrm{eff}}^{2}$. The effective mass $m_{\mathrm{eff}}=$ $E_{v} \sqrt{v_{v}^{2}-1} v_{v}^{-1}$ then grows linearly with the neutrino energy. (Natural units with $\hbar=c=\epsilon_{0}=1$ are used in this paper, yet we shall include explicit factors of $c$ when indicated by the context.) Indeed, at the time, a best fit to the available experimental neutrino mass data including the initial OPERA claim [11] suggested the conceivable existence of an "energydependent mass" of the neutrino, as evidenced in Figure 1 of [12]. The choice of the relation $E_{v}=|\vec{p}| v_{v}$ made in [8] was consistent with the need to model the initial OPERA claim [11] and is perfectly compatible with the concept of perturbative Lorentz breaking terms in the neutrino sector [9]. A Dirac-type equation leading to the Lorentznoncovariant dispersion relation used by Cohen and Glashow [8] can be obtained [9] from the current operator given in Eq. (2) of [13] upon a particular choice of $c^{\mu \nu}$ parameters in the generalized fermionic current operator (in the notation adopted in [13]). Then, assuming a constant neutrino speed $v_{v}>c$, one can effectively describe the apparent absence of 
energy dependence of the deviation of the neutrino speed from the speed of light $v_{v} \approx$ const. $\approx c$ (in the range $5 \mathrm{GeV}<$ $E_{\gamma}<50 \mathrm{GeV}$ ), according to the (falsified) initial claim made by OPERA [11], while remaining compatible with the framework of perturbative Lorentz breaking [13].

However, while there are advantages to assuming a Lorentz-noninvariant dispersion relation for superluminal neutrinos (such as the preservation of the timelike positive quantity $E_{v}^{2}-\vec{p}^{2}>0$ ), there are also a number of disadvantages. For example, if the dispersion relation $E_{\nu}=|\vec{p}| v_{\nu}$ holds in one particular Lorentz frame, then, under a Lorentz boost, in general, one has $E_{v}^{\prime} \neq\left|\vec{p}^{\prime}\right| v_{v}$ in the moving frame [8,9]. In order to illustrate the consequences of Lorentz noncovariance, let us consider a boost along positive $z$-axis into a frame which moves with velocity $u=c^{2} / v_{v}<c$. A particle moving along positive $z$-axis of the lab frame with four-momentum $p^{\mu}=\left(|\vec{p}| v_{v},|\vec{p}| \widehat{e}_{z}\right)$ is mapped onto $p^{\prime \mu}=\left(|\vec{p}| \sqrt{v_{v}^{2}-1}, \overrightarrow{0}\right)$ and thus is "at rest" in the moving frame. However, the general dispersion relation in the moving frame,

$$
E_{v}^{\prime}=-\frac{p_{z}^{\prime}}{2 v_{v}}-\frac{\left(p_{x}^{\prime 2}+p_{y}^{\prime 2}+p_{z}^{\prime 2}\right) v_{v}}{2 p_{z}^{\prime}} \quad\left(p_{z}^{\prime} \neq 0\right),
$$

is much more complicated. (Throughout this paper, we denote the spatial components of the four-vector $p^{\mu}=\left(E_{\gamma}, \vec{p}\right)$ by $\vec{p}$ and keep $|\vec{p}|$ explicitly in order to avoid confusion between $p^{2}=p^{\mu} p_{\mu}$ and $p^{2} \neq \vec{p}^{2}$.)

An alternative, commonly accepted dispersion relation for so-called tachyons (these are space-like, faster-than-light particles described by a Lorentz-invariant wave equation) reads as $E_{v}^{2}=\vec{p}^{2}-m_{v}^{2}$; that is, it is the "normal" dispersion relation with the negative sign of the mass square term (see $[2-7,14-23])$. Here, we calculate the threshold energy and the decay rate under the assumption of a Lorentz-invariant dispersion relation for the neutrino. We find that the alternate dispersion relation imposes tight restrictions on superluminality and has important phenomenological implications for neutrino masses.

\section{Dispersion Relations and Thresholds}

For tachyonic particles, starting from the pioneering work of Sudarshan et al. [14-16], continuing with the works of Feinberg [17, 18], and including the tachyonic neutrino hypothesis [2-6, 19-23], the following dispersion relation has been assumed for the tachyonic (space-like) solutions:

$$
\begin{aligned}
E_{\nu} & =\gamma_{\nu} m_{\nu}, \\
\left|\vec{p}_{\nu}\right| & =\gamma_{\nu} m_{\nu} v_{v}, \\
\left|\vec{p}_{\nu}\right| & =E_{\nu} v_{\nu}, \\
p^{\mu} p_{\mu} & =E_{\nu}^{2}-\vec{p}_{\nu}^{2}=-m_{\nu}^{2},
\end{aligned}
$$

where we use the suggestive subscript $v$ for "neutrino." These relations imply that $|\vec{p}|=E_{v} v_{v}$ instead of $E_{v}=|\vec{p}| v_{v}$. Here, the tachyonic Lorentz factor appears, which is $\gamma_{v}=$

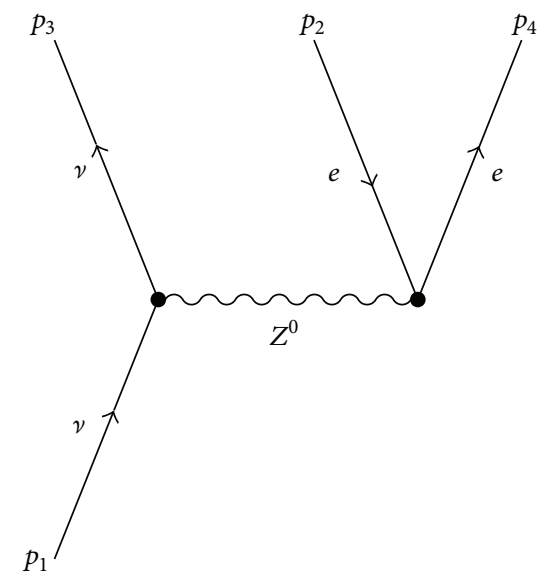

FIGURE 1: Conventions for tachyonic neutrino decay.

$1 / \sqrt{v_{v}^{2}-1}$. Tachyonic and tardyonic dispersion relations are unified upon assuming an imaginary value for $m$ in the tachyonic case (starting from the tardyonic case, one has $E=$ $m / \sqrt{1-v^{2}} \rightarrow \mathrm{i} m / \sqrt{1-v^{2}}=m / \sqrt{v^{2}-1}$, where the latter equation holds for tachyons). With the standard definitions of $\vec{p}$ and $E_{v}$, one has $\left|\vec{p}_{v}\right|=\gamma_{\nu} m v_{v}=E_{\gamma} v_{v}$ for both tardyons and tachyons.

In order to obtain the threshold energy for the LPCR decay $v \rightarrow e^{+} e^{-} v$, we use the following conventions (see Figure 1), inspired by Chap. 10 of [24], and define $E_{1}=$ $\sqrt{\vec{p}_{1}^{2}-m_{\nu}^{2}}$ and $E_{3}=\sqrt{\vec{p}_{3}^{2}-m_{\nu}^{2}}$ as the oncoming and outgoing neutrino energies, with $q=\left(E_{1}, \vec{p}_{1}\right)-\left(E_{3}, \vec{p}_{3}\right)$ being the fourmomentum of $Z^{0}$. Pair production threshold is reached for $q^{2}=4 m_{e}^{2}$ and $\cos \theta=\overrightarrow{p_{1}} \cdot \overrightarrow{p_{3}} /\left(\left|\vec{p}_{1} \| \vec{p}_{3}\right|\right)=1$. For collinear geometry, with all momenta pointing along $z$-axis, we have

$$
\begin{aligned}
q^{2} & =\left(\sqrt{p_{1 z}^{2}-m_{\nu}^{2}}-\sqrt{p_{3 z}^{2}-m_{\nu}^{2}}\right)^{2}-\left(p_{1 z}-p_{3 z}\right)^{2} \\
& =4 m_{e}^{2} .
\end{aligned}
$$

Furthermore, threshold obviously requires $E_{3}=0$. (This is possible for tachyonic particles, when $\left|\vec{p}_{3}\right|=p_{3 z}=m_{v}$. In this limit, the tachyonic particle becomes infinitely fast and loses all of its energy, which implies that it is impossible to detect it [25]. The counterintuitive loss of energy for tachyons under acceleration is a consequence of standard tachyonic kinematics $[2,6,7,14-18,26-28]$.$) When the relations E_{3}=0$ and $\left|\vec{p}_{3}\right|=p_{3 z}=m_{\nu}$ are substituted into (3), this yields

$$
p_{1 z}^{2}-m_{v}^{2}-\left(p_{1 z}-m_{v}\right)^{2}=4 m_{e}^{2} .
$$

Identifying $p_{1 z}=|\vec{p}|_{\mathrm{th}}$ with the threshold momentum, one easily finds

$$
|\vec{p}|_{\mathrm{th}}=\frac{2 m_{e}^{2}}{m_{v}}+m_{v}
$$

The threshold energy is then easily found as

$$
E_{\mathrm{th}}=\sqrt{\vec{p}_{\mathrm{th}}^{2}-m_{v}^{2}}=2 \frac{m_{e}}{m_{v}} \sqrt{m_{e}^{2}+m_{v}^{2}} \approx 2 \frac{m_{e}^{2}}{m_{v}} .
$$


Because we are using a tachyonic dispersion relation, the threshold energy can be expressed as a function of only the mass parameters. Larger tachyonic masses $m_{v}$ lead to lower threshold energies. In view of the tachyonic dispersion relation $m_{v}=E_{\text {th }} \sqrt{v_{\text {th }}^{2}-1}$, where $v_{\text {th }}$ is the neutrino velocity at threshold, we may convert the threshold energy into a function of the electron mass and the neutrino threshold velocity. For given $E_{v}$, the limit $m_{v} \ll m_{e}$ is equivalent to the limit $v_{\text {th }}^{2}-1=\delta_{\text {th }} \rightarrow 0$ because $m_{v}=E_{v} \sqrt{\delta_{\text {th }}}$. In this limit, we have

$$
E_{\mathrm{th}} \approx 2 \frac{m_{e}^{2}}{m_{v}}=2 \frac{m_{e}^{2}}{E_{\mathrm{th}} \sqrt{v_{\mathrm{th}}^{2}-1}} \Longrightarrow E_{\mathrm{th}} \approx \frac{\sqrt{2} m_{e}}{\left(v_{\mathrm{th}}^{2}-1\right)^{1 / 4}}
$$

Substituting the exact dispersion relation into the threshold condition $E_{\mathrm{th}}=2\left(m_{e} / m_{v}\right) \sqrt{m_{e}^{2}+m_{v}^{2}}$, and solving for $E_{\mathrm{th}}$, one obtains

$$
\begin{aligned}
E_{\mathrm{th}} & =\sqrt{2} m_{e}\left(1+\frac{v_{\mathrm{th}}}{\sqrt{v_{\mathrm{th}}^{2}-1}}\right)^{1 / 2} \\
& = \begin{cases}\frac{\sqrt{2} m_{e}}{\delta_{\mathrm{th}}^{1 / 4}} & \delta_{\mathrm{th}} \ll 1 \\
2 m_{e}+\frac{m_{e}}{4 \delta_{\mathrm{th}}} & \delta_{\mathrm{th}} \gg 1 .\end{cases}
\end{aligned}
$$

The exact expression (8) confirms (7) in the limit $\delta_{v} \ll$ 1 , which corresponds to the phenomenologically important limit of high-energy neutrinos. Smaller values of $\delta_{\text {th }}$ (approaching zero) correspond to smaller tachyonic neutrino masses and therefore to larger threshold energies. For given neutrino speed $v_{\text {th }}$, neutrinos with energy $E_{\text {th }}$ (or larger), under the hypothetical assumption of the tachyonic dispersion relation, have a tachyonic neutrino mass term large enough to make the decay via LPCR kinematically possible. Expressed differently, the tachyonic mass term $m_{v}=$ $E_{\text {th }} \sqrt{v_{\text {th }}^{2}-1}$ in this case is large enough to lead to LPCR decay at energy $E_{\text {th }}$, according to (6).

\section{Decay Rate and Timelike Noncovariant Dispersion Relation}

Given the complexities of calculating the decay rate due to LPCR using a tachyonic dispersion relation, it is extremely useful to first discuss the case of a Lorentz-noncovariant form $E_{v}=|\vec{p}| v_{v}$, using lab frame variables. For collinear incoming and outgoing neutrinos, threshold for pair production is reached at $q^{2}=\left(E_{1}-E_{3}\right)^{2}-\left(p_{1 z}-p_{3 z}\right)^{2}=\left(p_{1 z}-p_{3 z}\right)^{2}\left(v_{v}^{2}-1\right)=$ $4 m_{e}^{2}$, from which one derives (setting $\vec{p}_{3}=\overrightarrow{0}$ ) the following threshold values (in agreement with [8]):

$$
\begin{aligned}
& \left|\vec{p}_{1}\right|_{\text {th }}=\frac{2 m_{e}}{\sqrt{v_{v}^{2}-1}}, \\
& \left(E_{1}\right)_{\text {th }}=\frac{2 m_{e} v_{v}}{\sqrt{v_{v}^{2}-1}} .
\end{aligned}
$$

Here, $G_{F}$ is Fermi's coupling constant and $u$ and $v$ are the standard fundamental positive-energy and negative-energy bispinor solutions of the Dirac equation [29]. The invariant matrix element is

$$
\begin{aligned}
\mathscr{M}= & \frac{G_{F}}{\sqrt{2}}\left[\bar{u}\left(p_{3}\right) \gamma_{\lambda}\left(1-\gamma^{5}\right) u\left(p_{1}\right)\right] \\
& \times\left[\bar{u}\left(p_{4}\right)\left(c_{V} \gamma_{\lambda}-c_{A} \gamma_{\lambda} \gamma^{5}\right) v\left(p_{2}\right)\right] .
\end{aligned}
$$

Here, $c_{V} \approx 0$, and $c_{A} \approx-1 / 2$ [see Eq. (5.57) on p. 153 of [30]]. Following [9], we now make the additional assumption that the functional form of the projector sum over the spin orientations remains the same as for the ordinary Dirac equation even if the underlying dispersion relation is Lorentznoncovariant (for a general discussion on such models, see $[31,32])$. In this case, the sum over final state and the averaging over the initial spins leads to $(1 / 2) \sum_{\text {spins }}|\mathscr{M}|^{2}=$ $64 G_{F}^{2}\left(p_{1} \cdot p_{2}\right)\left(p_{3} \cdot p_{4}\right)$. This enters the lab frame expression for the decay rate [33]:

$$
\begin{aligned}
\Gamma & =\frac{1}{2 E_{1}} \int \frac{\mathrm{d}^{3} p_{3}}{(2 \pi)^{3} 2 E_{3}}\left(\int \frac{\mathrm{d}^{3} p_{2}}{(2 \pi)^{3} 2 E_{2}} \int \frac{\mathrm{d}^{3} p_{4}}{(2 \pi)^{3} 2 E_{4}}\right. \\
& \left.\times(2 \pi)^{4} \delta^{(4)}\left(p_{1}-p_{3}-p_{2}-p_{4}\right)\left[\frac{1}{2} \sum_{\text {spins }}|\mathscr{M}|^{2}\right]\right) \\
& =\frac{G_{F}^{2}}{12 \pi^{4}\left(2 E_{1}\right)} \int \frac{\mathrm{d}^{3} p_{3}}{2 E_{3}}\left(p_{1} \cdot p_{3} q^{2}\right. \\
& \left.+2\left(p_{1} \cdot q\right)\left(p_{3} \cdot q\right)\right),
\end{aligned}
$$

where $q=p_{1}-p_{3}$. The azimuthal symmetry suggests the use of cylindrical coordinates. The domain of integration contains, for given $p_{1}=\left(p_{1 z} v_{v}, 0,0, p_{1 z}\right)$, all permissible $\vec{p}_{3}=$ $p_{3 \rho} \widehat{e}_{\rho}+p_{3 z} \widehat{e}_{z}$, where $p_{3}^{\mu}=\left(\left|\vec{p}_{3}\right| v_{v}, \vec{p}_{3}\right)$. With $E_{v}=|\vec{p}| v_{v}$, the momentum transfer is

$$
q^{2}=-\left(p_{1 z}-p_{3 z}\right)^{2}-p_{3 \rho}^{2}+\left(p_{1 z}-\sqrt{p_{3 \rho}^{2}+p_{3 z}^{2}}\right)^{2} v_{v}^{2},
$$

where we require $q^{2}>4 m_{e}^{2} \approx 0$. Solving (12) for $p_{3 \rho}$, one obtains the boundary of the region of permissible $\vec{p}_{3}$ vectors. An example is given in Figure 2(a) in the form of a "sharpened ellipsoid" with "sharp" top near $p_{3 \rho} \rightarrow 0, p_{3 z} \rightarrow p_{1 z}$, and a "rounded" bottom with $p_{3 \rho} \rightarrow 0$, and $p_{3 z} \rightarrow-\left[\left(v_{v}-1\right) /\left(v_{v}+\right.\right.$ 1)] $p_{1 z}$. After somewhat tedious integration over the allowed $\vec{p}_{3}$ vectors, one obtains

$$
\begin{aligned}
\Gamma= & \frac{G_{F}^{2}}{2688 \pi^{3}} \frac{p_{1 z}^{5} \delta_{v}^{3}}{v_{v}} \approx \frac{1}{14} \frac{G_{F}^{2} E_{v}^{5} \delta_{v}^{3}}{192 \pi^{3}} \\
\frac{\mathrm{d} E_{v}}{\mathrm{~d} x} \approx & -\frac{G_{F}^{2}}{96 \pi^{4}\left(2 E_{v}\right)} \int_{q^{2}>0} \frac{\mathrm{d}^{3} p_{3}}{2 E_{3}}\left(E_{v}-E_{3}\right) \\
& \times\left[\left(p_{1} \cdot p_{3}\right) q^{2}+2\left(p_{1} \cdot q\right)\left(p_{3} \cdot q\right)\right] \\
= & -\frac{G_{F}^{2}}{86016 \pi^{3}} \frac{p_{1 z}^{6} \delta_{v}^{3}}{v_{v}} \approx-\frac{25}{448} \frac{G_{F}^{2} E_{v}^{6} \delta_{v}^{3}}{192 \pi^{3}},
\end{aligned}
$$




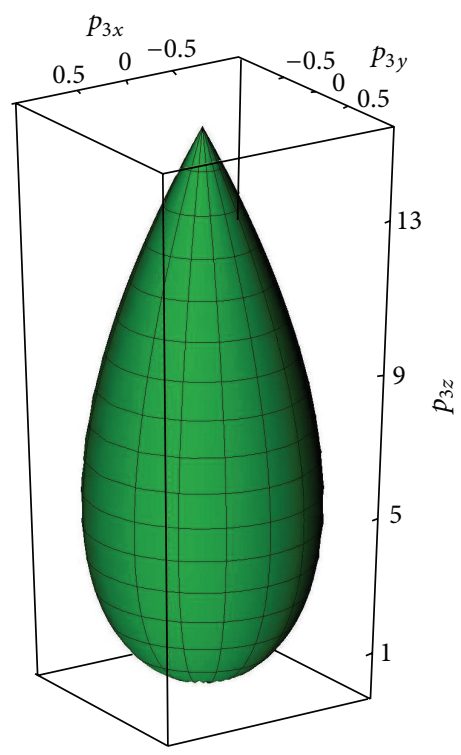

(a)

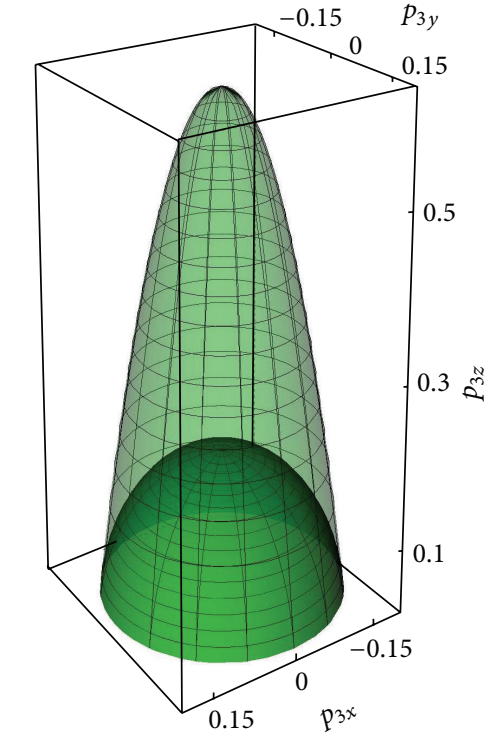

(b)

FIgURE 2: (a) Region of allowed outgoing momenta $\vec{p}_{3}$ for the decay of an incoming superluminal neutrino with $E_{v}=|\vec{p}| v_{v}$. The neutrino is incoming along positive $z$-axis $\left(p_{1 z}=15\right)$. The boundary of allowed $\vec{p}_{3}$ vectors constitutes a distorted ellipsoid with a "sharpened tip," obtained as a solution of setting $q^{2}=0$ in (12). (b) Region of allowed $\vec{p}_{3}$ vectors for an incoming tachyonic neutrino with $p_{1 z}=62$ and $-m_{v}^{2}=-(0.2)^{2}$, producing an electron-positron pair of mass $m_{e}=1$ (dispersion relation $E_{v}=\sqrt{\vec{p}_{\nu}^{2}-m_{\nu}^{2}}$ ). Final wave vectors $\left|\vec{p}_{3}\right|<m_{\nu}$ correspond to evanescent waves and are thus to be excluded [6].

for the energy loss per unit length, confirming the results given in Eq. (2) and Eq. (3) of [8] and in [9]. This confirmation of the results given in [8] (under the assumptions made in the cited paper, namely, the dispersion relation $E_{v}=|\vec{p}| v_{v}$ ), but using a different method, namely, phase-space integration directly in the laboratory frame, encourages us to apply the same method to the calculation of the tachyonic neutrino decay rate, where the use of the laboratory frame is indispensable. The confirmation also underlines the consistency of the theoretical formalism under a change of the assumptions made in the calculation.

\section{Decay Rate and Space-Like Covariant Dispersion Relation}

For an incoming tachyon, the particle state (space-like neutrino) may transform into an antiparticle state upon Lorentz transformation, and its trajectory may reverse the time ordering (see Figure 3). Thus, the interpretation of a tachyonic neutrino state as a particle or antiparticle may depend on the frame of reference, and we should calculate the process directly in the lab frame. The necessity to transform certain tachyonic particle field operators into antiparticle operators under Lorentz boosts has been stressed in $[6,17$, 18]. Incoming and outgoing states are required to be abovethreshold positive-energy states in the lab frame (causality and tachyonic trajectories are discussed in $[2,14-18]$ and Appendix A.2 of [34]).

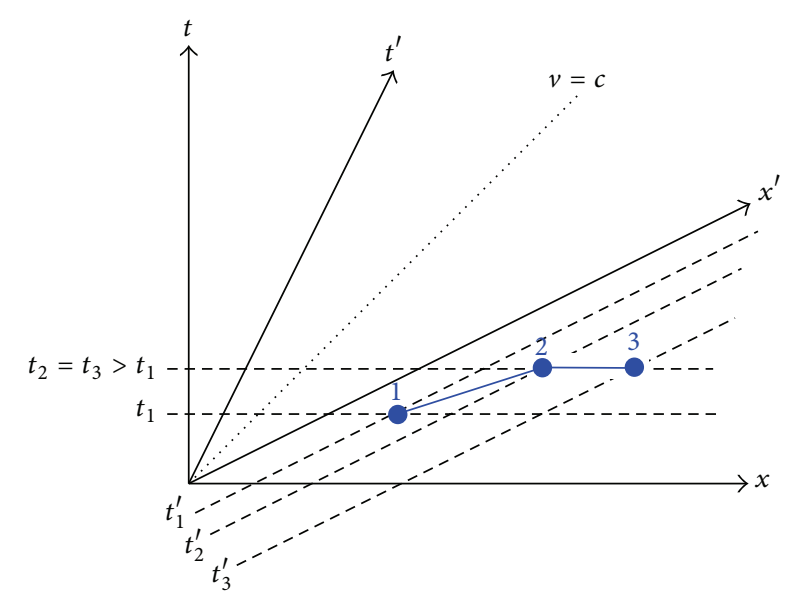

FIgURE 3: The world line $1 \mapsto 2 \mapsto 3$ describes the tachyonic neutrino decay into a zero-energy, infinitely fast neutrino. Complete reversal of the time ordering of the decay process takes place in the primed frame; the observer interprets the process as the decay of an incoming antineutrino along the trajectory $3 \mapsto 2 \mapsto 1$.

We consider the matrix element

$$
\begin{aligned}
\mathscr{M}= & \frac{G_{F}}{\sqrt{2}}\left[\bar{u}^{\mathscr{T}}\left(p_{3}\right) \gamma_{\lambda}\left(1-\gamma^{5}\right) u^{\mathscr{T}}\left(p_{1}\right)\right] \\
& \times\left[\bar{u}\left(p_{4}\right)\left(c_{V} \gamma^{\lambda}-c_{A} \gamma^{\lambda} \gamma^{5}\right) v\left(p_{2}\right)\right] .
\end{aligned}
$$


Here, $u^{\mathscr{T}}\left(p_{1}\right)$ and $u^{\mathscr{T}}\left(p_{3}\right)$ are Dirac spinor solutions of the tachyonic Dirac equation $[6,7]$. The bar denotes the Dirac adjoint. In the helicity basis (see Chap. 23 of [35] and [6, 7]), these are given by

$$
u_{ \pm}^{\mathscr{T}}(p)=\left(\begin{array}{c}
\sqrt{|\vec{p}| \pm m a_{ \pm}}(\vec{p}) \\
\pm \sqrt{|\vec{p}| \mp m a_{ \pm}}(\vec{p})
\end{array}\right),
$$

where $a_{ \pm}(\vec{p})$ are the fundamental helicity spinors (see p. 87 of [29]). Following $[6,7,19]$, we use the tachyonic sum rule of the fundamental tachyonic bispinor solutions [see Eq. (34a) of [6]]:

$$
\sum_{\sigma}(-\sigma) u_{\sigma}^{\mathscr{T}}(p) \otimes \bar{u}_{\sigma}^{\mathscr{T}}(p) \gamma^{5}=\not p-\gamma^{5} m
$$

where $p=(E, \vec{p})$ is the four-momentum and $\sigma$ is a helicity quantum number. We refer to $[6,7]$ for a thorough discussion; roughly speaking, factor $(-\sigma)$ in (16) restores the correct sign in the calculation of the time-ordered product of tachyonic field operators (the propagator) for the contribution of all virtual degrees of freedom of the tachyonic field [see Eqs. (46) $-(57)$ and Eq. (73)-(75) of [7]]. $\gamma^{5}$ matrix in (16) is a part of the natural Dirac "adjoint" for the tachyonic spinor. Namely, the adjoint equation to the tachyonic Dirac equation, $\left(\mathrm{i} \gamma^{\mu} \partial_{\mu}-\gamma^{5} m\right) \psi(x)=0$, reads as $\left[\bar{\psi}(x) \gamma^{5}\right]\left(\mathrm{i} \gamma^{\mu} \overleftarrow{\partial}_{\mu}-\gamma^{5} m\right) \psi(x)=$ 0 . As explained in Eqs. (73)-(75) of [17], right-handed particle states and left-handed antiparticle states (those with the "wrong" helicity) are excluded from the physical spectrum of the tachyonic field by a Gupta-Bleuler condition; these cannot contribute to the oncoming and outgoing neutrino states in Figure 1 [while they do contribute to the virtual states, that is, the propagator; see Eqs. (46)-(57) of [7]]. Both the incoming and the outgoing neutrinos in Figure 1 are real rather than virtual neutrinos. Hence, in order to calculate the LPCR decay rate, we use the modified sum over tachyonic spinors:

$$
\sum_{\sigma} u_{\sigma}^{\mathscr{T}}(p) \otimes \bar{u}_{\sigma}^{\mathscr{T}}(p)=\left(1+\gamma^{5} \not \hat{p}\right)\left(\not p-\gamma^{5} m_{\nu}\right) \gamma^{5}
$$

where $\tau=(1,0,0,0)$ is a timelike unit vector, $\widehat{p}=\vec{p} /|\vec{p}|$ is the unit vector in $\vec{p}$ direction, and upon promotion to a fourvector, we have $\widehat{p}^{\mu}=(0, \widehat{p})$, so that $1+\gamma^{5} t \vec{p}=1-\vec{\Sigma} \cdot \vec{p} /|\vec{p}|$ becomes a left-handed helicity projector.

We thus calculate with an incoming, positive-energy, lefthelicity tachyonic neutrino. One obtains the modified sum over spins $\widetilde{\sum}_{\text {spins }}$ in the matrix element:

$$
\begin{aligned}
& \sum_{\text {spins }}|\mathscr{M}|^{2}=\frac{G_{F}^{2}}{2} \operatorname{Tr}\left[\frac{1}{2}\left(1+\gamma^{5} \not p_{3}\right)\left(\not p_{3}-\gamma^{5} m_{\nu}\right)\right. \\
& \cdot \gamma^{5} \gamma_{\lambda}\left(1-\gamma^{5}\right) \frac{1}{2}\left(1+\gamma^{5} \not p_{1}\right)\left(\not p_{1}-\gamma^{5} m_{\nu}\right) \\
& \left.\cdot \gamma^{5} \gamma_{\nu}\left(1-\gamma^{5}\right)\right] K^{\lambda \rho} .
\end{aligned}
$$

Here, $K^{\lambda \rho}=\operatorname{Tr}\left[\left(\not p_{4}+m_{e}\right)\left(c_{V} \gamma^{\lambda}-c_{A} \gamma^{\lambda} \gamma^{5}\right)\left(\not p_{2}+m_{e}\right)\left(c_{V} \gamma^{\rho}-\right.\right.$ $\left.\left.c_{A} \gamma^{\rho} \gamma^{5}\right)\right]$ is the familiar trace from the outgoing fermion pair. The decay rate is given by (11) under the replacement $(1 / 2) \sum_{\text {spins }}|\mathscr{M}|^{2} \rightarrow \widetilde{\sum}_{\text {spins }}|\mathscr{M}|^{2}$. The integrals over the momenta of the outgoing fermion pair $\left(\mathrm{d}^{3} p_{2}\right.$ and $\left.\mathrm{d}^{3} p_{4}\right)$ are done using $\left(p_{2}^{2}=p_{4}^{2}=m_{e}^{2}\right)$. Consider

$$
\begin{aligned}
& J_{\lambda \rho}(q)=\int \frac{\mathrm{d}^{3} p_{2}}{2 E_{2}} \int \frac{\mathrm{d}^{3} p_{4}}{2 E_{4}} \delta^{(4)}\left(q-p_{2}-p_{4}\right)\left(p_{2 \lambda} p_{4 \rho}\right) \\
& =\frac{\pi}{24} \sqrt{1-\frac{4 m_{e}^{2}}{q^{2}}}\left[g_{\lambda \rho}\left(q^{2}-4 m_{e}^{2}\right)\right. \\
& \left.+2 q_{\lambda} q_{\rho}\left(1+\frac{2 m_{e}^{2}}{q^{2}}\right)\right] .
\end{aligned}
$$

It remains to analyze the domain of allowed $\vec{p}_{3}$ vectors [see the "cupola structure" in Figure 2(b)], which is defined by the requirement $q^{2}>4 m_{e}^{2}$, for $p_{1}^{\mu}=\left(\sqrt{p_{1 z}^{2}-m_{\nu}^{2}}, 0,0, p_{1 z}\right)$. The dispersion relation $E_{v}=\sqrt{\vec{p}_{\nu}^{2}-m_{v}^{2}}$ implies that

$$
q^{2}=2\left(\sqrt{E_{1}^{2}+m_{v}^{2}} \sqrt{E_{3}^{2}+m_{v}^{2}} \cos \theta-E_{1} E_{3}-m_{v}^{2}\right) .
$$

Here, $\theta$ is the polar angle in spherical coordinates:

$$
p_{3}^{\mu}=\left(E_{3},\left|\vec{p}_{3}\right| \sin \theta \cos \varphi,\left|\vec{p}_{3}\right| \sin \theta \sin \varphi,\left|\vec{p}_{3}\right| \cos \theta\right) .
$$

Pair production threshold is reached, for given $E_{1}$ and $E_{3}$, by solving (20) for $u=\cos \theta$, setting $q^{2}=4 m_{e}^{2}$. After somewhat tedious integration over the allowed $\vec{p}_{3}$ vectors (no masses can be neglected), one obtains

$$
\Gamma= \begin{cases}\frac{G_{F}^{2} m_{v}^{6}}{128 \pi^{3} m_{e}^{2}} \frac{\left(E_{v}-E_{\mathrm{th}}\right)^{2}}{E_{\mathrm{th}}} & E_{v} \gg E_{\mathrm{th}} \\ \frac{G_{F}^{2} m_{v}^{4}}{288 \pi^{3}} E_{v} & E_{v} \gg E_{\mathrm{th}},\end{cases}
$$

for the decay rate, and

$$
\frac{\mathrm{d} E_{v}}{\mathrm{~d} x}= \begin{cases}\frac{G_{F}^{2} m_{v}^{5}}{64 \pi^{3}} \frac{\left(E_{v}-E_{\mathrm{th}}\right)^{2}}{E_{\mathrm{th}}} & E_{v} \gg E_{\mathrm{th}} \\ \frac{G_{F}^{2} m_{v}^{4}}{144 \pi^{3}} E_{1}^{2} & E_{v} \gg E_{\mathrm{th}},\end{cases}
$$

for the energy loss rate. In the high-energy limit, one may (somewhat trivially) rewrite the expressions as follows $\left(m_{v}=\right.$ $\left.E_{1} \sqrt{\delta_{\gamma}}\right)$ :

$$
\begin{gathered}
\Gamma=\frac{G_{F}^{2} E_{v}^{5} \delta_{v}^{2}}{288 \pi^{3}}, \\
\frac{\mathrm{d} E_{v}}{\mathrm{~d} x}=\frac{G_{F}^{2} E_{v}^{6} \delta_{v}^{2}}{144 \pi^{3}}, \\
E_{v} \gg E_{\mathrm{th}} .
\end{gathered}
$$

These results confirm that it is possible to use the tachyonic bispinor formalism [2-7] for the calculation of decay rates of tachyonic particles. 


\section{Constraints on the Mass of a Tachyonic Neutrino}

Our threshold relation (8) is based on a Lorentz-covariant dispersion relation. Only neutrinos with $E_{v}<E_{\text {th }}=$ $\sqrt{2} m_{e} / \delta_{\text {th }}^{1 / 4}$ survive the possibility of generalized leptonic Čerenkov radiation over a sufficiently long path length. The hypothetical observation of an absence of neutrinos above some energy $E_{\text {th }}$ could thus be interpreted as a constraint on the neutrino mass. Let us assume a neutrino mass of $m_{v}=$ $X \mathrm{eV}$, where $X$ is generally assumed to be of order unity or less. Then, threshold is reached for $m_{v}=X \mathrm{eV}, \delta_{\text {th }}=3.67 \times$ $10^{-24} X^{4}$, and $E_{\text {th }}=(522 / X) \mathrm{GeV}$.

The IceCube experiment [36, 37] has observed 37 neutrinos having energies $E_{v}>10 \mathrm{TeV}$ during 3 years of data taking. Three of these events had energies $E_{v}>1 \mathrm{PeV}$, and one (often referred to as "Big Bird") had $E_{v}=(2.004 \pm 0.236) \mathrm{PeV}$. According to the IceCube collaboration [37], the spectrum of the 37 neutrinos is well fitted by a slope $\sim E_{\gamma}^{-2}$, which includes astrophysical as well as background atmospheric neutrinos, the latter being exclusively below $0.4 \mathrm{PeV}$. However, their best fit to the spectrum predicts 3.1 additional events for $E_{v}>$ $2 \mathrm{PeV}$, and yet none were seen. Preliminary data for the fourth year includes 17 additional events, with none seen for $E_{\gamma}>$ $1 \mathrm{PeV}$ [38]. These facts suggest to the IceCube authors [36, 37] the possibility that there may be a cutoff for the spectrum for neutrinos above $E \approx 2 \mathrm{PeV}$. The hypothesis is given further support by models which show that the Glashow resonance [39] (resonant $\bar{\nu}_{e} e^{-} \rightarrow W^{-} \rightarrow$ anything) should add between zero and three times the number of events that appear in the interval $1 \mathrm{PeV}<E_{v}<2 \mathrm{PeV}$ as part of a broad peak centered around 6.3 PeV [40]. While evidence for the cutoff is disputed and alternative explanations have been proposed [41], the significance of such a cutoff has been analyzed in the light of superluminal neutrinos [42, 43].

Let us add a few clarifying remarks here. First, we note that the plots in the paper [37] refer to the neutrino flux as a function of neutrino energy; the events were apparently sufficiently well reconstructed so that no excess neutrino energy in addition to the energy deposited inside the detector is expected. Our Figure 4 is based on Figure 4 of [37]. Meanwhile, members of the IceCube collaboration have presented preliminary evidence for a throughgoing muon of energy $\geq$ $(2.6 \pm 0.3) \mathrm{PeV}$ which could be interpreted as a decay product of a neutrino of even higher energy [44, 45]. If the throughgoing muon could indeed be assigned to an ultra-high-energy neutrino of nonatmospheric origin, then it would push the conceivable cutoff seen by IceCube to even higher energies, further constraining the tachyonic mass term of the relevant neutrino flavor. So far, the authors of [37] (see the right column on page 4 of [37]) observe that "this [the lack of highenergy events] may indicate, along with the slight excess in lower energy bins, either a softer spectrum or a cutoff at high energies."

Assuming $E_{\mathrm{th}} \approx 2 \mathrm{PeV}$ we would find using (8) that $\delta_{\mathrm{th}}=$ $\left(\sqrt{2} m_{e} / E_{\mathrm{th}}\right)^{4} \approx 1.7 \times 10^{-38}$, and we would, furthermore, find that $m_{v}=\sqrt{\delta_{\text {th }}} E_{\text {th }} \approx 0.00026 \mathrm{eV}$ (i.e., $-m_{v}^{2} \approx-6.8 \times 10^{-8} \mathrm{eV}^{2}$ ) for one or more of the three neutrino flavors (conceivably,

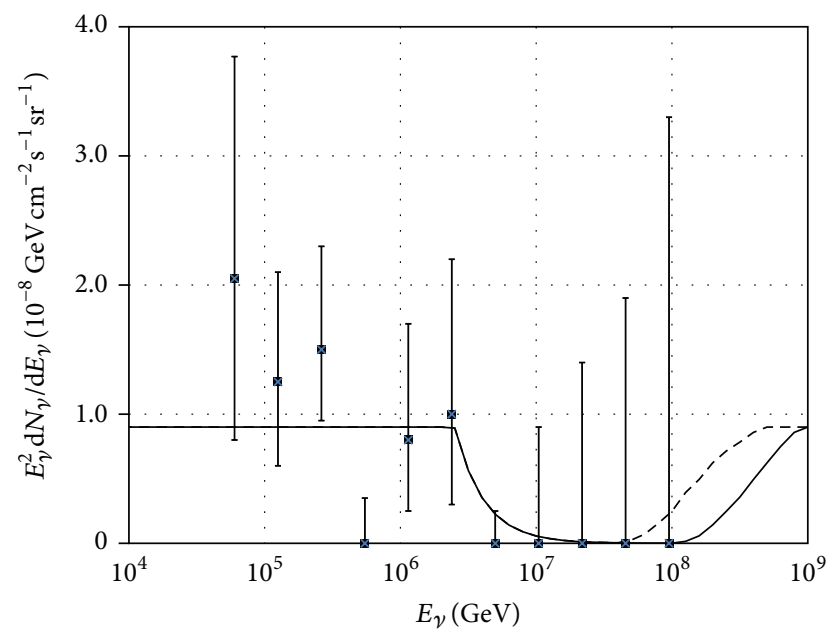

FIgURE 4: Three years of data from the IceCube experiment showing flux $\mathrm{d} N_{v} / \mathrm{d} E_{v}$ multiplied by $E_{v}^{2}$ plotted against the neutrino energy $E_{v}$. The solid and dashed curves show what would be expected for $E_{\gamma}^{-2}$ power law for the flux, with $E_{\text {th }}=2.5 \mathrm{PeV}$ threshold, and two arbitrarily assumed values for the source distance, $L$ (dashed curve) and $L / 2$ (solid curve). The drop to zero above $E_{\text {th }}$ only occurs for those neutrino flavors having a tachyonic mass consistent with a 2.5 PeV threshold.

the one with the smallest absolute value of $m_{v}^{2}$ ). A shifted cutoff $[44,45]$ of $E_{\mathrm{th}} \approx 3 \mathrm{PeV}$ would be consistent with a tachyonic neutrino mass of $m_{v}=0.00017 \mathrm{eV}$. One might object that it is not possible to have one (or more) tachyonic flavor masses $\left(m^{2}<0\right)$ and satisfy both neutrino oscillation data and the recent findings from cosmology for the sum of the flavor masses; that is, $\Sigma m \approx 0.32 \mathrm{eV}[46,47]$. However, such consistency can be achieved using 3 active-sterile $\pm m^{2}$ (tardyon-tachyon) neutrino pairs [48]. The curves in Figure 4 were generated using an assumed pure $E_{\gamma}^{-2}$ power law for flux $N$ beyond the assumed threshold, $E_{\text {th }}$. We then use our $\mathrm{d} E_{v} / \mathrm{d} x$ formula (22b) for $E_{v}>E_{\text {th }}$ to find the modified $N E_{v}^{2}$ spectrum. Good agreement is found with the IceCube data at a threshold $E_{\text {th }}=2.5 \mathrm{PeV}$, although much more statistics will be needed to determine if the cutoff is real.

\section{Conclusions}

Three main conclusions of the current investigation can be drawn. (i) As described in Section 2, the assumption of a Lorentz-covariant, tachyonic dispersion relation leads to tight bounds on conceivable tachyonic neutrino mass terms, for whatever neutrino flavor is causing the possible $2 \mathrm{PeV}$ cutoff. The tachyonic decay rate due to LPCR is most conveniently calculated in the laboratory frame because of the spacelike kinematics involved in the process, which leads to a nonunique time ordering of the trajectories, as discussed in Section 4. (ii) We may apply the formalism of the tachyonic bispinor solutions of the tachyonic Dirac equation [2-5] recently developed in $[6,7,19]$ to the calculation of the tachyonic neutrino decay, as outlined in Section 4. (iii) A comparison of recent IceCube data with the results for the 
calculated tachyonic decay rates reveals that a tachyonic neutrino could possibly explain a possible sharp cutoff in IceCube data but only if the neutrino flavor involved has a very specific tachyonic mass. In a more general context, the calculation of tachyonic thresholds and decay rates based on Lorentzcovariant dispersion relations could be of phenomenological significance for string theories, some of which predict the existence of tachyons $[49,50]$. The same is true for the precise calculation of the tail of the beta decay spectrum, which is influenced by a conceivably tachyonic neutrino mass term [51].

\section{Competing Interests}

The authors declare that there are no competing interests regarding the publication of this paper.

\section{Acknowledgments}

This research has been supported by the National Science Foundation (Grant no. PHY-1403973).

\section{References}

[1] V. L. Dadykin, G. T. Zatsepin, V. B. Karchagin et al., "Detection of a rare event on 23 February 1987 by the neutrino radiation detector under Mont Blanc,", JETP Letters, vol. 45, pp. 593-595, 1987, Pis'ma ZhETF, vol. 45, pp. 464, 1987.

[2] A. Chodos, A. I. Hauser, and V. A. Kostelecký, "The neutrino as a tachyon," Physics Letters B, vol. 150, no. 6, pp. 431-435, 1985.

[3] T. Chang, "A new Dirac-type equation for tachyonic neutrinos," https://arxiv.org/abs/hep-th/0011087.

[4] T. Chang, "Parity violation and neutrino mass," Nuclear Science and Techniques/Hewuli, vol. 13, no. 3, pp. 129-133, 2002.

[5] T. Chang, "Parity violation and a preferred frame," http://arxiv .org/abs/quant-ph/0204002vl.

[6] U. D. Jentschura and B. J. Wundt, "Localizability of tachyonic particles and neutrinoless double beta decay," European Physical Journal C, vol. 72, no. 2, pp. 1-13, 2012.

[7] U. D. Jentschura and B. J. Wundt, "From generalized dirac equations to a candidate for dark energy," ISRN High Energy Physics, vol. 2013, Article ID 374612, 21 pages, 2013.

[8] A. G. Cohen and S. L. Glashow, "Pair creation constrains superluminal neutrino propagation," Physical Review Letters, vol. 107, no. 18, Article ID 181803, 2011.

[9] F. Bezrukov and H. M. Lee, "Model dependence of the bremsstrahlung effects from the superluminal neutrino at OPERA," Physical Review D, vol. 85, no. 3, Article ID 031901, 2012.

[10] P. C. W. Davies and I. G. Moss, "Cosmological bounds on tachyonic neutrinos," Astroparticle Physics, vol. 35, no. 10, pp. 679680, 2012.

[11] T. Adam, N. Agafonova, A. Aleksandrov et al., "The influence of Earth rotation in neutrino speed measurements between CERN and the OPERA detector," 2011, http://arxiv.org/abs/1109.4897.

[12] F. Tamburini and M. Laveder, "Apparent Lorentz violation with superluminal Majorana-tachyonic neutrinos at OPERA?" Physica Scripta, vol. 85, no. 3, Article ID 035101, 2012.
[13] V. A. Kostelecky and R. Lehnert, "Stability, causality, and Lorentz and CPT violation," Physical Review D, vol. 63, Article ID 065008, 2001.

[14] O. M. P. Bilaniuk, V. K. Deshpande, and E. C. G. Sudarshan, "'Meta' relativity," American Journal of Physics, vol. 30, pp. 718723, 1962.

[15] J. Dhar and E. C. G. Sudarshan, "Quantum field theory of interacting tachyons," Physical Review, vol. 174, no. 5, pp. 1808$1815,1968$.

[16] O.-M. Bilaniuk and E. C. G. Sudarshan, "Causality and spacelike signals," Nature, vol. 223, no. 5204, pp. 386-387, 1969.

[17] G. Feinberg, "Possibility of faster-than-light particles," Physical Review, vol. 159, no. 5, pp. 1089-1105, 1967.

[18] G. Feinberg, "Lorentz invariance of tachyon theories," Physical Review D, vol. 17, no. 6, pp. 1651-1660, 1978.

[19] U. D. Jentschura and B. J. Wundt, "Neutrino helicity reversal and fundamental symmetries," Journal of Physics G: Nuclear and Particle Physics, vol. 41, no. 7, Article ID 075201, 2014.

[20] R. Ehrlich, "Implications for the cosmic ray spectrum of a negative electron neutrino (mass) ${ }^{2}$," Physical Review D, vol. 60, no. 1, Article ID 017302, 4 pages, 1999.

[21] R. Ehrlich, "Is there a $4.5 \mathrm{PeV}$ neutron line in the cosmic ray spectrum?" Physical Review D-Particles, Fields, Gravitation and Cosmology, vol. 60, Article ID 073005, 1999.

[22] R. Ehrlich, "Evidence for two neutrino mass eigenstates from SN 1987A and the possibility of superluminal neutrinos," Astroparticle Physics, vol. 35, no. 10, pp. 625-628, 2012.

[23] R. Ehrlich, "Tachyonic neutrinos and the neutrino masses," Astroparticle Physics, vol. 41, pp. 1-6, 2013.

[24] D. Griffiths, Introduction to Elementary Particles, John Wiley \& Sons, New York, NY, USA, 1987.

[25] See the neutrino cross sections tabulated at http://cupp.oulu.fi/ neutrino/nd-cross.html.

[26] E. Recami, "Superluminal waves and objects: an overview of the relevant experiments," Journal of Physics: Conference Series, vol. 196, Article ID 012020, 2009.

[27] O. M. Bilaniuk, “Tachyons," Journal of Physics: Conference Series, vol. 196, no. 1, Article ID 012021, 2009.

[28] S. K. Bose, "Aspects of Tachyon theory," Journal of Physics: Conference Series, vol. 196, Article ID 012022, 2009.

[29] C. Itzykson and J. B. Zuber, Quantum Field Theory, McGrawHill, New York, NY, USA, 1980.

[30] J. Horejsi, Fundamentals of Electroweak Theory, Karolinum Press, Prague, Czech Republic, 2002.

[31] G. Rubtsov, P. Satunin, and S. Sibiryakov, "Calculation of cross sections in Lorentz-violating theories," Physical Review D, vol. 86, no. 8, Article ID 085012, 2012.

[32] V. A. Kostelecky and M. Mewes, "Neutrinos with Lorentzviolating operators of arbitrary dimension," Physical Review D, vol. 85, no. 9, Article ID 096005, 2012.

[33] M. John, "Phase space and decay rates," in Particle Basics, pp. $1-8,2011$.

[34] U. D. Jentschura, D. Horváth, S. Nagy, I. Nándori, Z. Trócsányi, and B. Ujvári, "Weighing the neutrino," International Journal of Modern Physics E, vol. 23, no. 1, Article ID 1450004, 2014.

[35] V. B. Berestetskii, E. M. Lifshitz, and L. P. Pitaevskii, "Quantum electrodynamics," in The Course on Theoretical Physics, vol. 4, Pergamon Press, Oxford, UK, 2nd edition, 1982.

[36] M. G. Aartsen, R. Abbasi, Y. Abdou et al., "First observation of PeV-energy neutrinos with icecube," Physical Review Letters, vol. 111, Article ID 021103, 2013. 
[37] M. G. Aartsen, M. Ackermann, J. Adams et al., "Observation of high-energy astrophysical neutrinos in three years of icecube data," Physical Review Letters, vol. 113, no. 10, Article ID 101101, 2014.

[38] O. Botner, "IceCube Neutrino Observatory: present and future," in Proceedings of the IceCube Particle Astrophysics Symposium, Madison, Wis, USA, May 2015.

[39] S. L. Glashow, "Resonant scattering of antineutrinos," Physical Review, vol. 118, no. 1, pp. 316-317, 1960.

[40] V. Barger, L. Fu, J. G. Learned, D. Marfatia, S. Pakvasa, and T. J. Weiler, "Glashow resonance as a window into cosmic neutrino sources," Physical Review D, vol. 90, Article ID 121301, 2014.

[41] O. Kalashev and S. Troitsky, "IceCube astrophysical neutrinos without a spectral cutoff and 1015-1017 eV cosmic gamma radiation," JETP Letters, vol. 100, no. 12, pp. 761-765, 2015.

[42] F. W. Stecker and S. T. Scully, "Propagation of superluminal PeV IceCube neutrinos: a high energy spectral cutoff or new constraints on Lorentz invariance violation," Physical Review D, vol. 90, no. 4, Article ID 043012, 2014.

[43] F. W. Stecker, "Limiting superluminal electron and neutrino velocities using the $2010 \mathrm{Crab}$ Nebula flare and the IceCube PeV neutrino events," Astroparticle Physics, vol. 56, pp. 16-18, 2014.

[44] S. Schoenen and L. Raedel, "Detection of a multi-PeV neutrinoinduced muon event from the Northern sky with IceCube," 2015, http://www.astronomerstelegram.org/?read=7856.

[45] S. S. Schoenen and L. Raedel, 2015, https://www.icrr.u-tokyo.ac .jp/indico/getFile.py/access? contribId=43\&sessionId=6/\&resId $=0 \&$ materialid $=$ slides\& confld $=23$.

[46] R. A. Battye and A. Moss, "Evidence for massive neutrinos from cosmic microwave background and lensing observations," Physical Review Letters, vol. 112, Article ID 051303, 2014.

[47] J. Hamann and J. Hasenkamp, "A new life for sterile neutrinos: resolving inconsistencies using hot dark matter," Journal of Cosmology and Astroparticle Physics, vol. 2013, no. 10, article 044, 2013.

[48] R. Ehrlich, "Six observations consistent with the electron neutrino being a $m^{2}=-0.11 \pm 0.02 \mathrm{eV}^{2}$ tachyon," Astroparticle Physics, vol. 66, pp. 11-17, 2015.

[49] J. Polchinski, String Theory (Volume 1): An Introduction to the Bosonic String, Cambridge University Press, Cambridge, UK, 1998.

[50] J. Polchinski, String Theory (Volume 2): Superstring Theory and Beyond, Cambridge University Press, Cambridge, UK, 1998.

[51] J. Ciborowski, "Hypothesis of tachyonic neutrinos," Acta Physica Polonica B, vol. 29, pp. 113-121, 1998. 

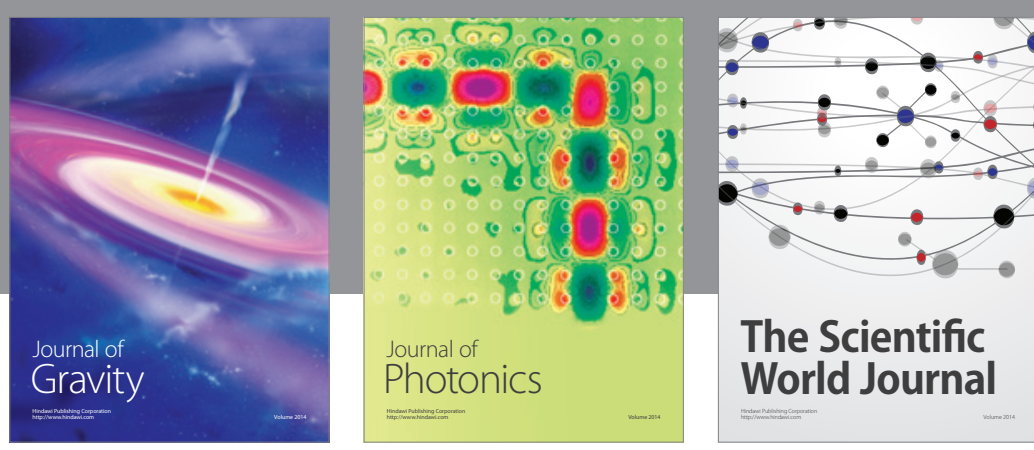

The Scientific World Journal
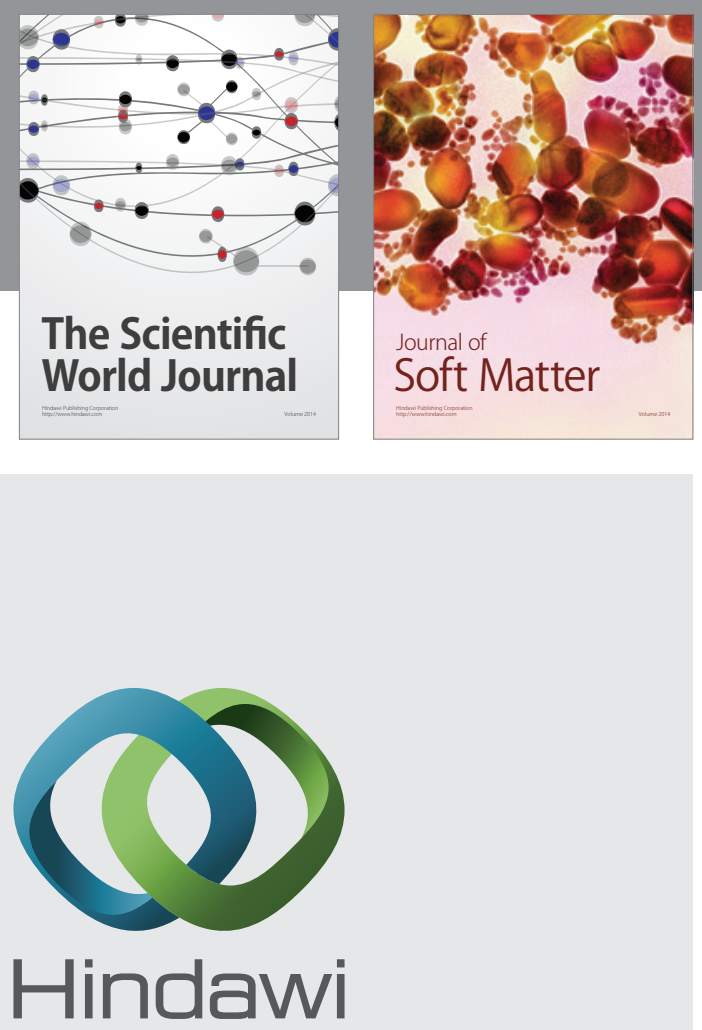

Submit your manuscripts at

http://www.hindawi.com

nternational Journal of

Statistical Mechanics
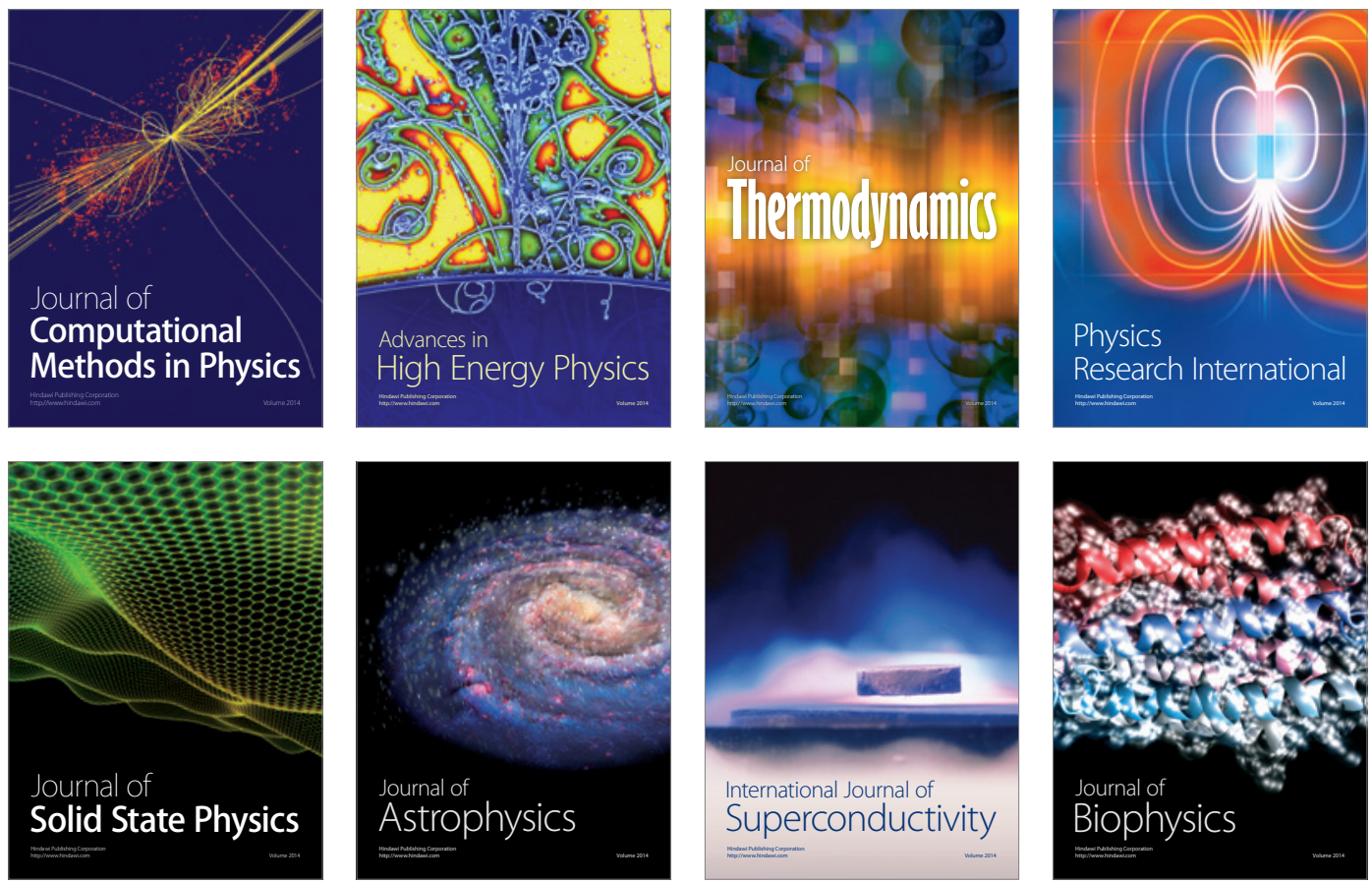
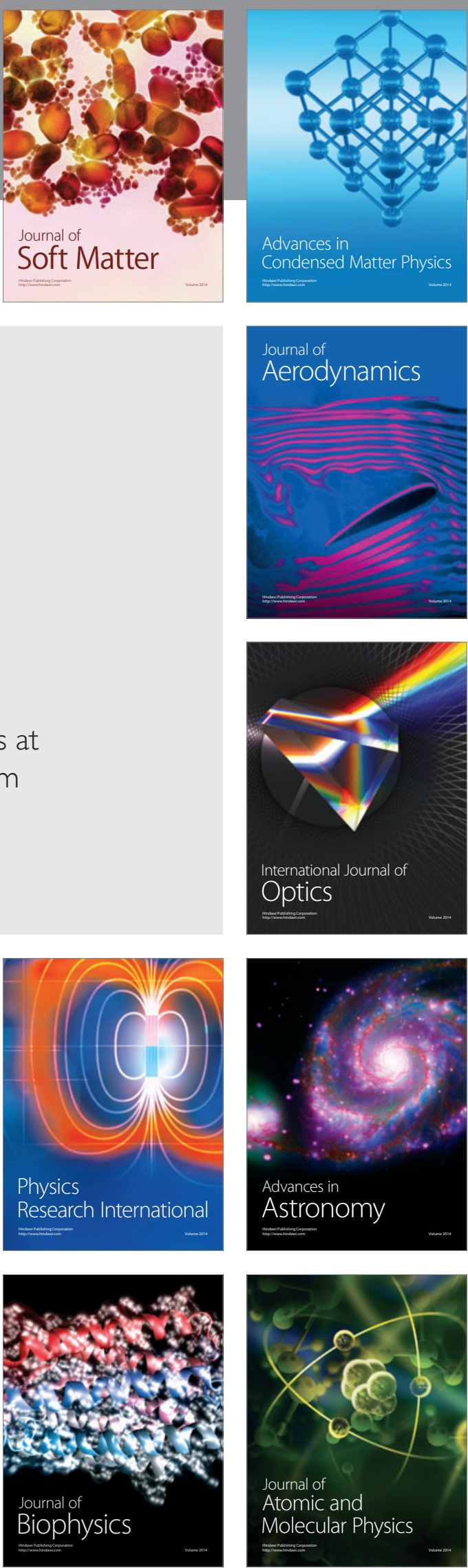